\title{
Numerical simulation of wave spectrum during a typhoon
}

\author{
Congying $\mathrm{KONG}^{1,2,3, \mathrm{a}} \mathrm{Hao} \mathrm{LIU}^{4}$ \\ ${ }^{1}$ Tianjin Port Engineering Institute Co., Ltd. of CCCC First Harbor Engineering Co., Ltd., Tianjin, 300222,China \\ ${ }^{2} \mathrm{CCCC}$ First Harbor Engineering Company Ltd., Tianjin, China \\ ${ }^{3}$ Key Laboratory of Coastal Engineering Hydrodynamic, CCCC, Tianjin, 300222, China \\ ${ }^{4}$ Tianjin Nangang Industrial Zone Water Supply Co. Ltd, Tianjin, 300000, China
}

\begin{abstract}
The third-generation wave model WAVEWATCH-III was used to numerically simulate the wave under the influence of a typhoon in the coastal area of China. The wave spectrum at the buoy point was output, and the characteristics of the wave spectrum were analyzed. The change of the wave spectrum during the typhoon process reflected the growth process of typhoon formation, development and extinction. The relationship between the wave spectrum and the wind direction was intuitively shown by the directional spectrum, indicating the coexistence of wind waves and swells in the sea area during the typhoon process.
\end{abstract}

\section{Introduction}

Wave spectrum is an important statistical property of random waves. It not only contains the second-order information of waves, but also gives the distribution of wave energy relative to frequency and direction. It is the most effective way to describe waves. At present, the wave spectrum has been widely used in the strength and stability calculation of various offshore platforms. Compared with the characteristic parameters such as wave height and period, the wave spectrum can more effectively reflect the internal structure of the wave field. At the same time, each wave element can be obtained by each order spectral moment. The study of wave spectrum is of great significance for the construction and maintenance of marine engineering, and it is also an important basis for the study of wave prediction, coastal sediment movement and offshore hydraulic structure $\operatorname{design}^{[1]}$.

Modern wave observation mainly relies on the wave meter, but large-scale long-term sequence of sea observation, the price is expensive, and in extreme weather ( cold wave, typhoon ) operability is not strong, so the use of wave numerical simulation method to study the time and spatial distribution of waves, to make up for the lack of measured wave data has become an effective way. The wave spectrum obtained by numerical simulation can be used for the prediction of large-area waves, the diffraction and refraction of waves, the force and vibration of hydraulic structures, the response of hull, buoy and other floating bodies to waves, and the study of sediment movement ${ }^{[2]}$.

\section{Model Setting and Verification}

The WAVEWATCH- III model for large-scale wave simulation in the full spectral space jointly developed by the U.S Atmospheric and Oceanic Administration and the U.S Environmental Prediction Center is based on the solution of the spectral action balance equation ${ }^{[3]}$.

Within WAVEWATCH-III, the basic spectrum is the wavenumber-direction spectrum $\mathrm{F}(\mathrm{k}, \theta)$, which has been selected because of its invariance characteristics with respect to physics of wave growth and decay for variable water depths. The output, however, consists of the more traditional frequency-direction spectrum $F(f r, \theta)$. The different spectra can be calculated from $F(k, \theta)$ using straightforward Jacobian transformations.

In a general sense, wave action $\mathrm{A}=E / \sigma$ is conserved(Whitham, 1965; Bretherthon and Garrett, 1968). This makes the wave action density spectrum $\mathrm{N}(\mathrm{k}, \theta) \equiv \mathrm{F}(\mathrm{k}, \theta) / \sigma$ the spectrum of choice within the model. Wave propagation then is described by $D N / D t=S / \sigma$

Where $D / D t$ represents the total derivative (moving with a wave component) and $\mathrm{S}$ represents the net effect of sources and sinks for the spectrum $\mathrm{F}$.

The study area is $110^{\circ} \mathrm{E} \sim 139^{\circ} \mathrm{E}, 13^{\circ} \mathrm{N} \sim 40^{\circ} \mathrm{N}$, and the terrain data are derived from the ETOPO1; The wind field adopts CCMP wind field with spatial resolution of $0.25^{\circ} \times 0.25^{\circ}$ and time interval of $6 \mathrm{~h}$. The model was calculated from June 21 to 27 , and the results were output at a 1 hour interval. The measured data come from buoy 1 and buoy2. Typhoon path map and buoy position are shown in Figure 1.

Figure 2 is the comparison between the calculated

\footnotetext{
*Corresponding author: ${ }^{\text {*k} k o n g c o n g y i n g @ t p e i . c o m . c n ~}$
} 
and measured significant wave height at the buoy site. From the effective wave height process curve, the measured wave height is in good agreement with the calculated wave height, which can accurately reflect the trend of wave size. The correlation coefficient $\mathrm{R}$ between the calculated values and the measured values of buoy 1 and buoy 2 were 0.956 and 0.971 , respectively, and the root mean square error RMSE were 0.331 and 0.368 , respectively. From the data, the model calculation results are reasonable and credible.

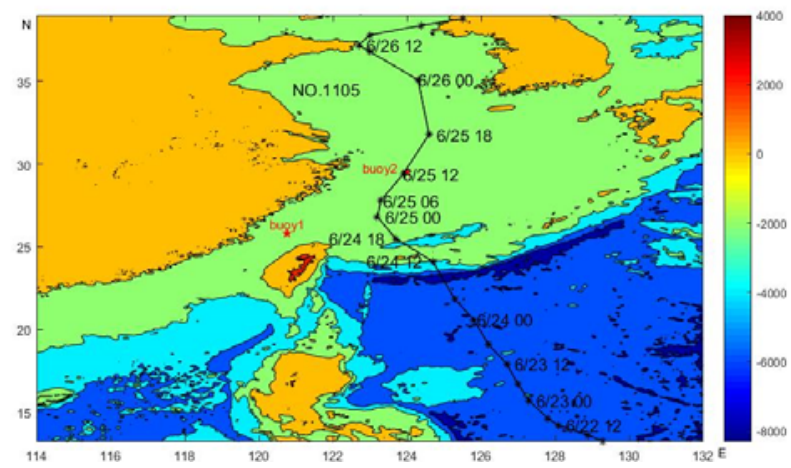

Figure1 Typhoon path map and buoy position
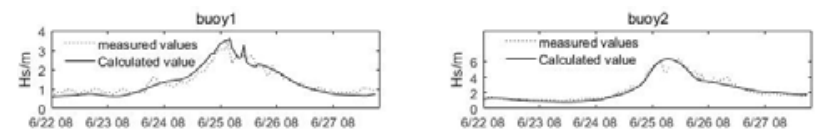

Figure2 the comparison between the calculated and measured significant wave height at the buoy site

\section{3 results and analysis}

\subsection{One-dimensional spectrum characteristics analysis}

Figs. 3 and 4 represent the one-dimensional wave spectrum, and Fig. 5 shows the spectral peak corresponding to the period with large typhoon influence. From figures 3 and 4, the wave spectrum is a single peak spectrum at $12: 00$ on 23 th, which indicates that the wave field is a single wind wave or surge, and the peak frequency at each buoy point is above $0.15 \mathrm{~Hz}$. Typhoon 1105 on 24th and 25th is in the growth period. Affected by the sea surface wind, the energy of wind waves and swells increases, and the energy of waves gradually moves to the low frequency part, and the frequency of spectral peaks is less than $0.1 \mathrm{~Hz}$. On 26 and 27 days, the typhoon intensity gradually weakened, the spectral peak significantly decreased, the spectral shape gradually expanded, and the spectral peak frequency shifted to the right.

In Fig5, the maximum spectral peak of buoyl was $45 \mathrm{~m}^{2} \cdot \mathrm{s}$ at $4: 00$ on $25 \mathrm{th}$, and the peak frequency was $0.0453 \mathrm{~Hz}$. The buoy2 also reached the maximum spectral peak of $52.8 \mathrm{~m}^{2} \cdot \mathrm{s}$ at 4:00 on $25 \mathrm{th}$, and the peak frequency was $0.073 \mathrm{~Hz}$; This is because 24 and 25 days are the strongest periods of typhoon development. From the comparison of typhoon path in Figure 1, the buoy is close to the typhoon center during this period, and the buoys are in an open ocean with sufficient energy growth. Fig 5 shows that the spectral peak first increases and then decreases, which exactly reflects the growth process of typhoon formation, development and extinction.

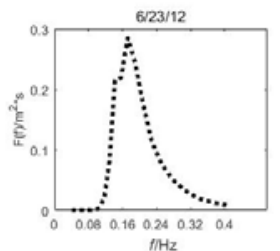

142

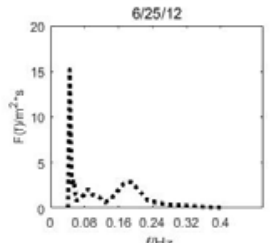

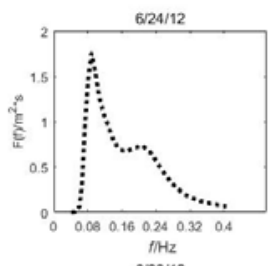

$6 / 26 / 12$

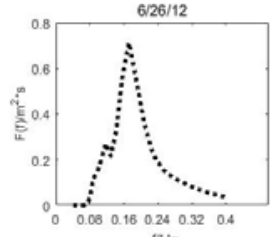

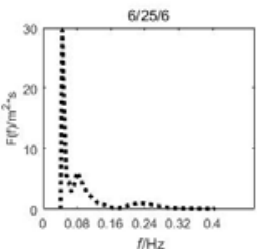

$6 / 27 / 0$

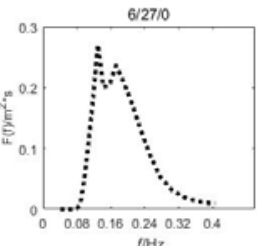

Figure 3 one-dimensional wave spectrum at buoy 1
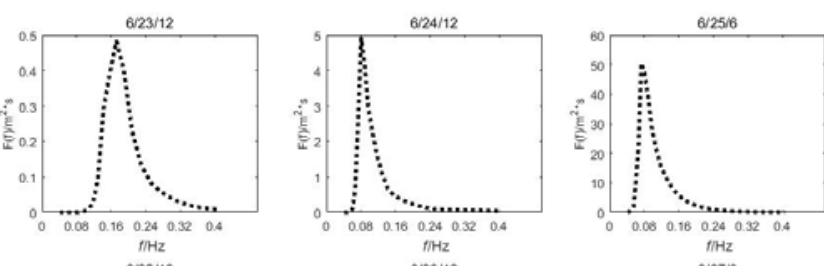

$3 / 26 / 12$
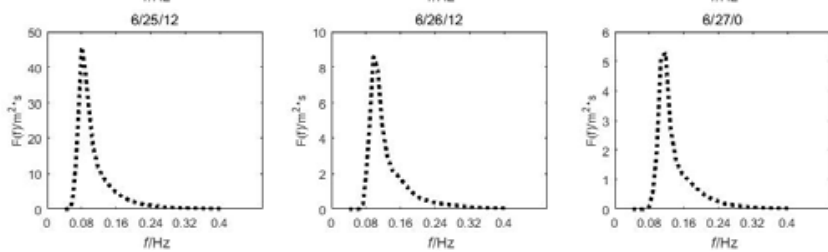

Figure4 one-dimensional wave spectrum at buoy2
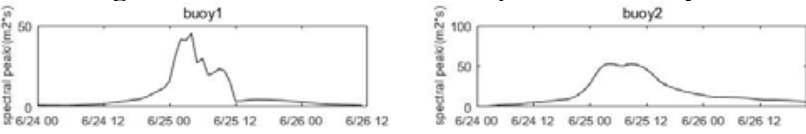

Figure5 the spectral peak corresponding to the period with large typhoon influence

\subsection{Analysis of directional spectrum characteristics}

The spectrum that describes the composition of waves along different directions is called directional spectrum. Directional spectrum is a function of frequency and direction, which can describe the distribution of wave energy relative to frequency and direction, and some statistical characteristics of wave space.

$$
\mathrm{S}(\omega, \theta)=\mathrm{S}(\omega) \mathrm{D}(\omega, \theta)
$$

$\mathrm{S}(\omega)$ is frequency spectrum; $D(\omega, \theta)$ reflects a function of energy relative to the direction distribution, $\theta$ is the angle between the constituent wave and the main wave direction ${ }^{[4]}$.

Figs 6 11 are directional spectra at six different times. The radius of the circle in the graph represents the frequency $(\mathrm{Hz})$, and the scale on the arc is the direction ( degree). Directional spectrum value is expressed in color stripes. And arrows represent wind direction. 

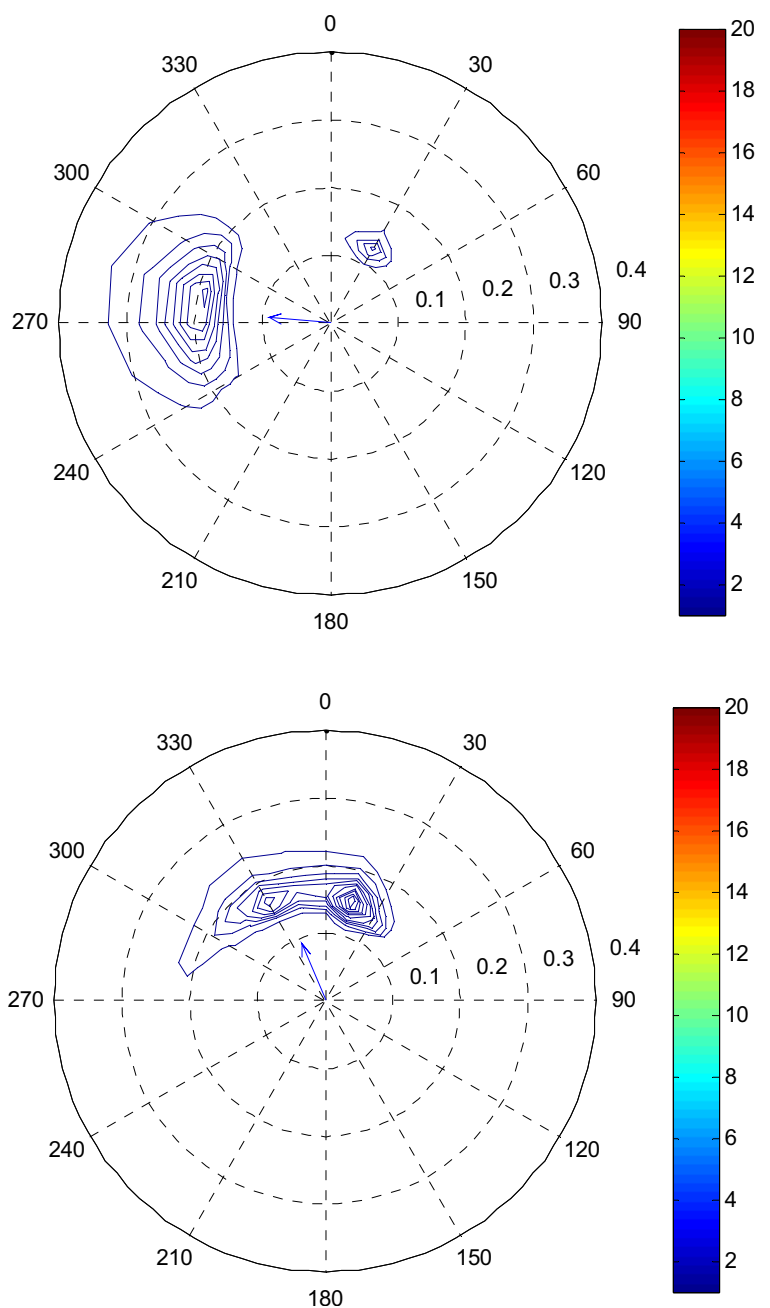

Figure6 Directional spectrum of buoys 1 and buoy2 varies with frequency and direction at 12:00 on 23rd

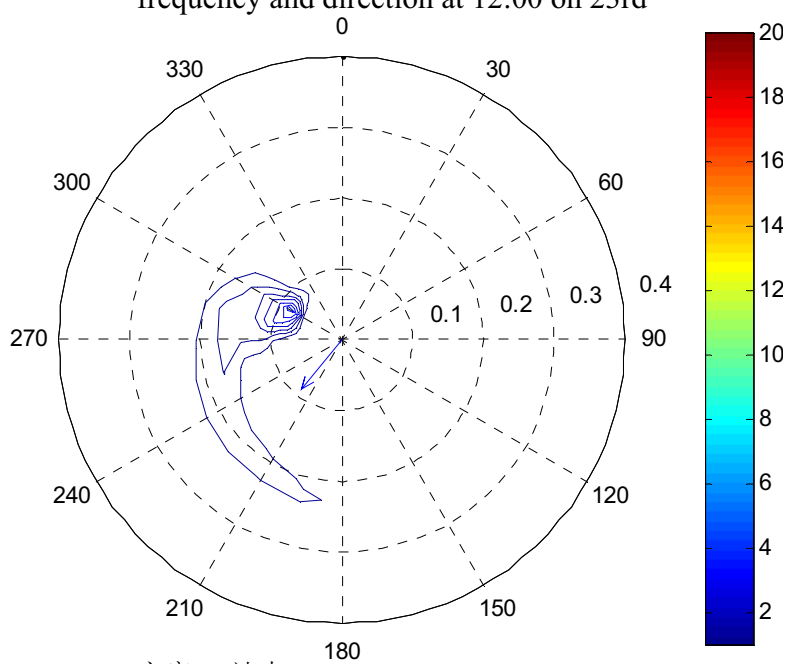

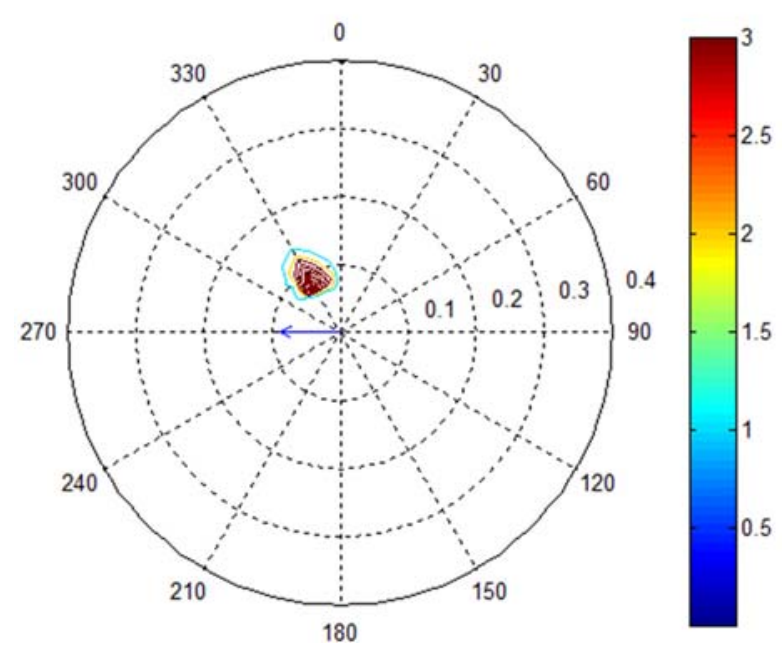

Figure7 Directional spectrum of buoys 1 and buoy 2 varies with frequency and direction at 12:00 on 24rd
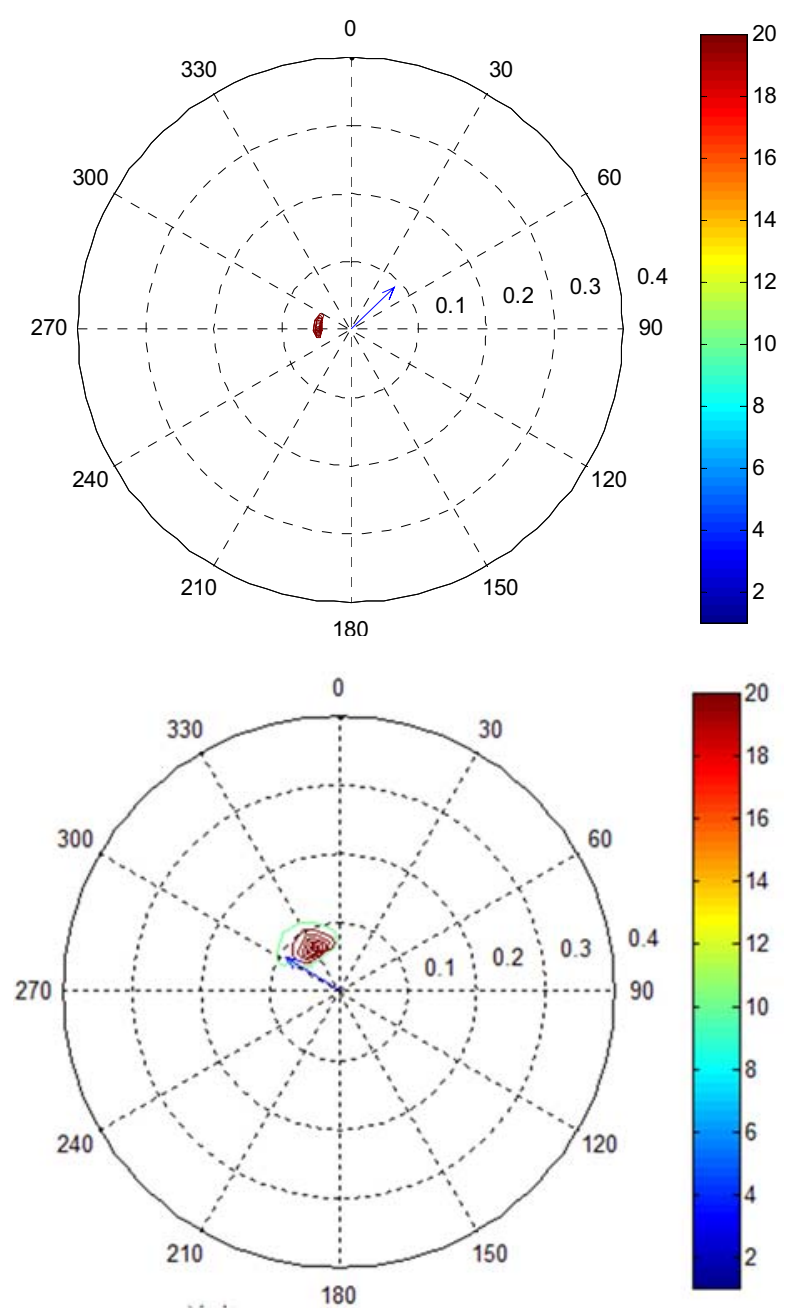

Figure8 Directional spectrum of buoys 1 and buoy2 varies with frequency and direction at 06:00 on $25 \mathrm{rd}$ 


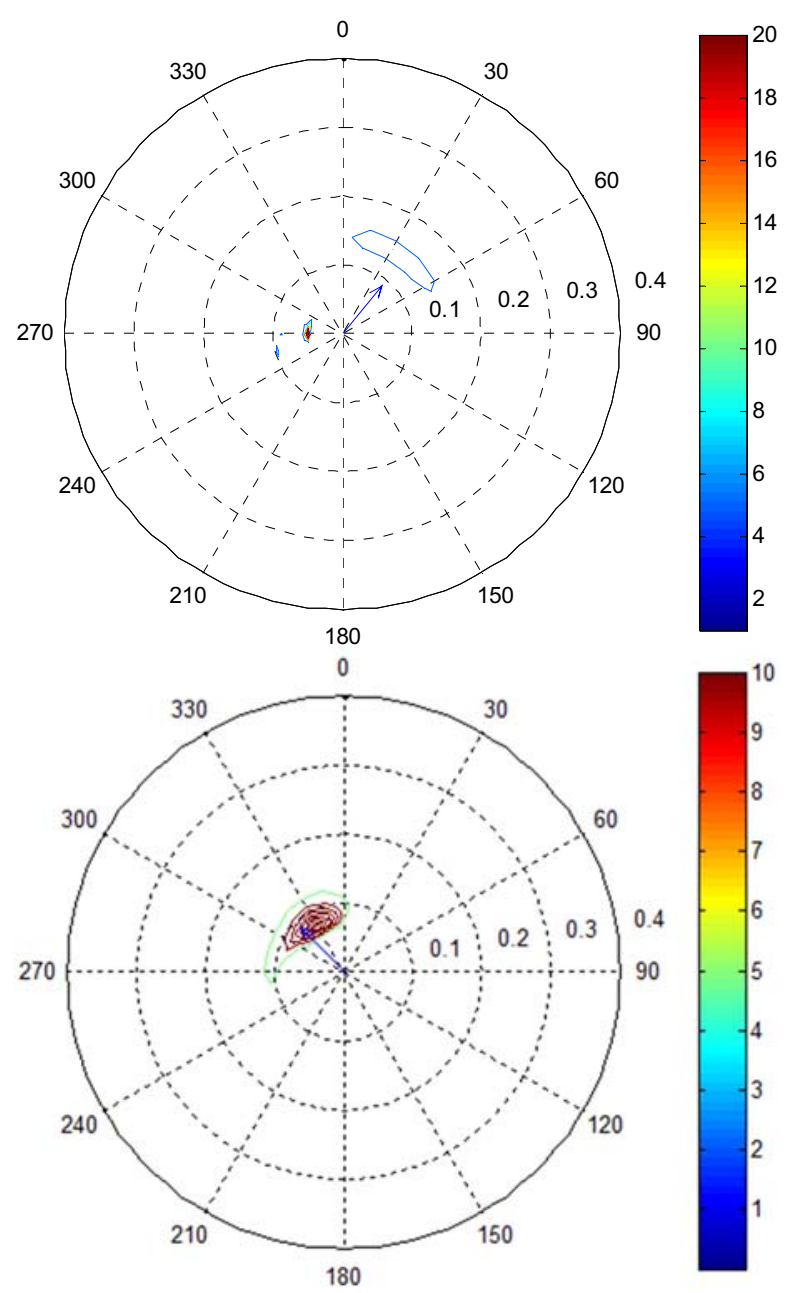

Figure9 Directional spectrum of buoys 1 and buoy2 varies with frequency and direction at 12:00 on 25rd

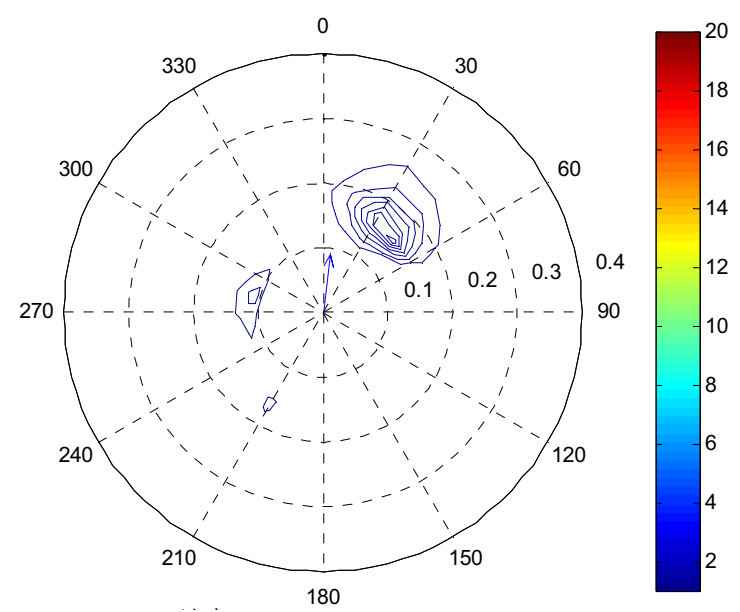

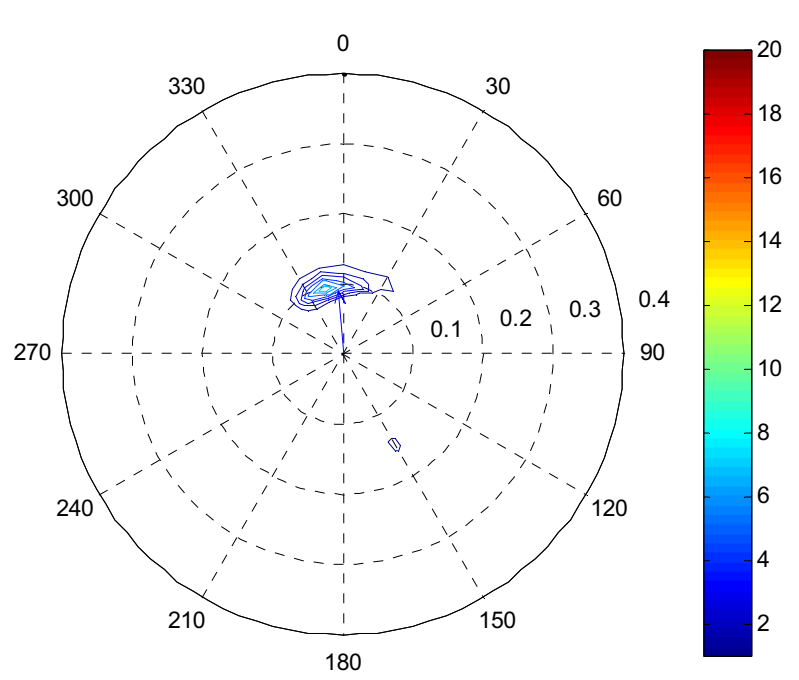

Figure10 Directional spectrum of buoys 1 and buoy 2 varies with frequency and direction at 12:00 on 26rd
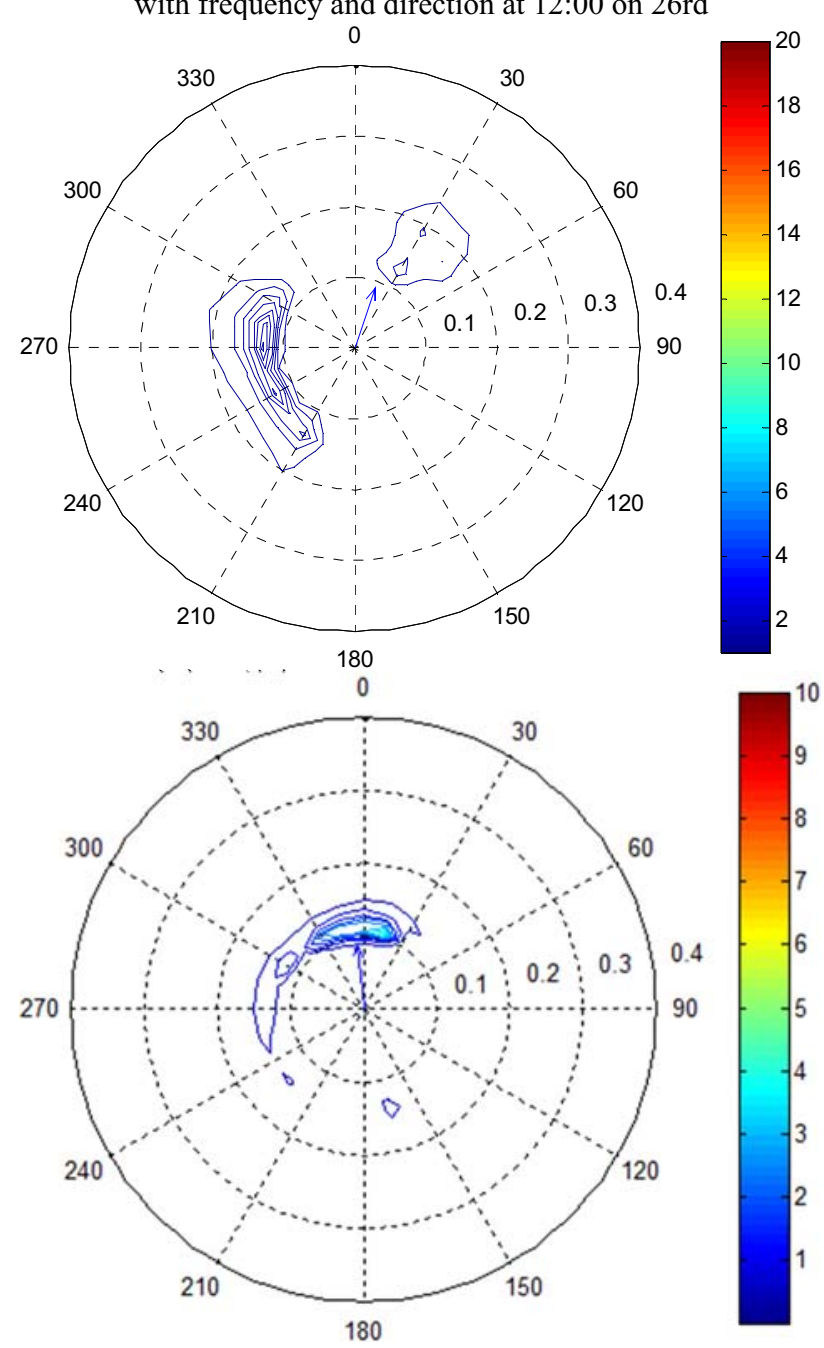

Figure11 Directional spectrum of buoys 1 and buoy 2 varies with frequency and direction at 00:00 on $27 \mathrm{rd}$

At 12:00 ( Figure 6 ) on 23rd, it can be found that the frequency range of the spectrum is between $0.15 \mathrm{~Hz}$ and $0.3 \mathrm{~Hz}$, the frequency range is wide, and the direction span is also large. The above characteristics show that the wave energy is more dispersed at this time, which is corresponding to the one-dimensional spectral characteristics of this time. With the increase of typhoon 
wind speed in the sea area from 24rd to 25rd (Fig7 $\sim$ Fig9), the energy of buoy points increased, and the energy of wave spectrum reached the maximum (the color strip represents the directional spectrum value).

In Figs. 6 11, the direction of the wave spectrum energy concentration of buoy 1 is inconsistent with the wind direction, while the place of the wave spectrum energy concentration of buoy 2 is gradually consistent with the wind direction, which reflects the relative position relationship between the typhoon center path and the two buoys : the direction of the typhoon movement is far from buoy 1 , and towards buoy 2 .

Taking the two-dimensional spectrum of buoy 1 at each time as an example, part of the wave spectrum energy in the figure is concentrated between $250^{\circ}$ and $300^{\circ}$, and another part of the energy changes with the wind direction. The propagation direction of swell in the wave is not affected by the wind, and the propagation direction of wind wave changes with the wind direction. Therefore, it indicates that there are wind waves and swells in the sea area at the same time. When the angle between the two is large, the direction separation of wind waves and swells occurs ( the part pointing to the arrow and the part far from the arrow in the figure ), which exactly reflects the bimodal spectrum of one-dimensional spectrum at each time in Figs.3 $4^{[5]}$.

\section{Conclusions}

WAVEWATCH-III model can well respond to the change process of typhoon waves in the sea area, and the model calculation results are reasonable and reliable. One-dimensional frequency spectrum reflects the typhoon from growth to extinction, with the increase of the severity of the sea, the peak frequency of the wave spectrum moves to the left, and the energy is concentrated in the low frequency part ; In the decay process, the spectral peak gradually decreases, and the peak frequency tends to move to high frequency.

The directional spectrum shows the distribution of waves in various directions and frequency ranges ; The relationship between the direction of energy propagation and the wind direction is more intuitive to show the coexistence of wind waves and swells in the typhoon process and the characteristics of wind waves and swells. The obtained wave spectrum plays an important guiding role in air-sea interaction, upper ocean dynamics, wave prediction, ocean remote sensing and ocean engineering.

\section{References}

[1] Kong Congying.2013. Numerical simulation of typhoon waves around the waters of China's offsea J. Marine Environmental Science, Vol. 32 No. 3, P. 419-423.

[2] YANG Shengqiang. 2015.Observed typhoon wave spectrum in northern South China Sea. J. Chinese Journal of Oceanology and Limnology,Vol. 33 No. 5, P. 1286-1294.

[3] Hendrik L. Tolman. 2009.User manual and system documentation of WAVEWATCH III. Environmental Modeling Center Marine Modeling and Analysis Branch,the U.S.

[4] ZHANG teng. 2016. A Comparative Study of Stationary Waves Spectrum Analysi. D. Dalian University of Technology.

[5] YANG zhong-liang.2017.The wave characteristics and the analysis of wave spectrum off the southeastern coast of Zhaitang Island. D. Journal of Marine Sciences. 